\title{
Retro-orbital nocardiosis masquerading as a neoplasm- A case report
}

\author{
Prashant Mule ${ }^{1, *}$, Rohini Kelkar ${ }^{2}$, Sanjay Biswas ${ }^{3}$, Vivek Bhat ${ }^{4}$ \\ ${ }^{1}$ Senior Resident, ${ }^{2} \mathrm{HOD},{ }^{3,4}$ Professor, Dept. of Microbiology, Tata Memorial Hospital, Mumbai, India
}

*Corresponding Author:

Email: prashant.mule88@gmail.com

\begin{abstract}
Nocardiosis caused by an aerobic gram positive, weakly acid fast actinomycete is a localized or disseminated infection. The disease usually affects patients with defective cell mediated immunity. These infections are infrequent but pose a great challenge in terms of diagnosis and treatment as the treatment is prolonged and relapse is known to occur. The incidence has been increasing recently which can be attributed to the improvements in diagnostic facilities and increased prevalence of patients with HIV, chronic alcoholism, diabetes mellitus, patients receiving aggressive antineoplastic chemotherapy, long standing corticosteroid therapy and patients undergoing organ transplantation with immunomodulator drugs. Nocardiosis has been described in patients with underlying chronic pulmonary diseases. Primary nocardial infection includes pulmonary (60-70\%), brain, musculoskeletal system and cutaneous or subcutaneous lesions. Disseminated disease is defined by the nocardial involvement in two or more organs. Nocardiosis associated with HIV usually appears in patients with advanced disease with CD4 counts less than 50 cells $/ \mathrm{mm}^{3}$. Sulfonamides like trimethoprim-sulfamethoxazole are the drugs of choice for nocardiosis and are successfully used for treatment. Nocardiosis has been described to mimic tuberculosis, aspergillosis, bronchogenic and metastatic lung carcinomas, brain and musculoskeletal tumours. Here we describe an unusual presentation of retro-orbital nocardiosis masquerading as a neoplasm which was successfully treated with cotrimoxazole and ceftriaxone.
\end{abstract}

Keywords: Nocardiosis, Actinomycete, Antineoplastic drugs, HIV, Cotrimoxazole.

\section{Introduction}

Nocardia, the gram-positive, branched, filamentous aerobic actinomycete is an uncommon human pathogen that infects lungs (most common), skin and subcutaneous tissues, central nervous system (CNS), muskuloskeletal system or other organs. Defective cell mediated immunity is the most important risk factor for nocardiosis especially for disseminated infection. It can present as localized or disseminated infections depending on the cellular immunity of patient. ${ }^{1,2}$ Nocardia species are ubiquitous in the environment and are found worldwide in soil and decaying organic matter. Nocardiosis is associated with chronic diseases like diabetes mellitus, alcoholism, bronchogenic and metastatic lung cancers and alveolar proteinosis. ${ }^{3}$ The mode of acquisition of organism is by inhalation into the respiratory tract or direct inoculation into the skin, mucosal sites or at the site of traumatic injury. Nosocomial transmission is very rare ${ }^{4}$. Lungs are the common site affected with nocardiosis is able to produce infection in a host compromised by systemic disease or anti-inflammatory therapy (50\%); however, it can also produce infection in patients with no concurrent abnormality. Colonization or subclinical infection occurs, especially within the respiratory tract of patients with chronic obstructive pulmonary disease (COPD), malignancy and metastatic lung cancers, bronchial asthma, bronchiectasis and tuberculosis. There is relatively sparse data on the incidence of nocardiosis. The increase in incidence may be attributed to increase in the number of immunosuppressed patients like HIV, cancer, and patients undergoing organ transplantation receiving high dose chemotherapeutic drugs. There is also high index of clinical suspicion and improved diagnostic capacity of microbiology laboratories. ${ }^{5,6} \mathrm{We}$ describe an unusual presentation of post traumatic retro-orbital nocardiosis with compromised immune status masquerading as a neoplasm. Nocardiosis has been described to mimic lung cancer, tuberculosis, aspergillosis and metastatic tumors in the brain.

\section{Case Report}

A 22 year old women presented with complaints of pain and swelling over the left fronto-temporal region and fever since 5-6 months. Patient revealed a history of road traffic accident which took place 1.5 years ago. The swelling was gradually increased over the left fronto-temporal region, left lateral canthus and around upper and lower eye lid region. On ophthalmic examination, vision was normal (Right Eye- 6/6, Left Eye-6/18) with restricted movement of eye and jaw on left side. The left eye was displaced antero-inferiorly showing conjunctival chemosis with purulent discharge. Complete blood count showed $\mathrm{Hb} 10.2 \mathrm{gm} / \mathrm{dl}$ and WBC count 11100 cells $/ \mathrm{mm}^{3}$ neutrophils $70 \%$, lymphocyte $26 \%$, eosinophil 4\%. Hepatitis b surface antigen (HbsAg) and HCV antibodies were non-reactive. HIV antibodies were reactive which was further confirmed with HIV-I RNA PCR. Patient was referred to integrated counseling and testing center (ICTC) for further management and viral load estimation and CD4 count. Magnetic resonance imaging of the face and orbit showed large ill-defined lobulated soft tissue intensity lesion in left fronto-temporal region with intra-orbital extension suggestive of neoplastic etiology and provisionally diagnosed as plexiform neurofibroma. Based on the radiological findings, patient was referred 
to Tata memorial hospital for evaluation and further line of management in view of neoplastic etiology. CT guided fine needle aspiration cytology was performed. The pus was aspirated and sent for histopathological examination to rule out malignant etiology and the specimen was also sent for microbiological examination. Gram stain of the pus showed long gram positive bacilli in branching filamentous pattern. Modified Kinyoun's acid fast stain with $1 \% \mathrm{H}_{2} \mathrm{SO}_{4}$ showed long acid fast bacilli with branching.

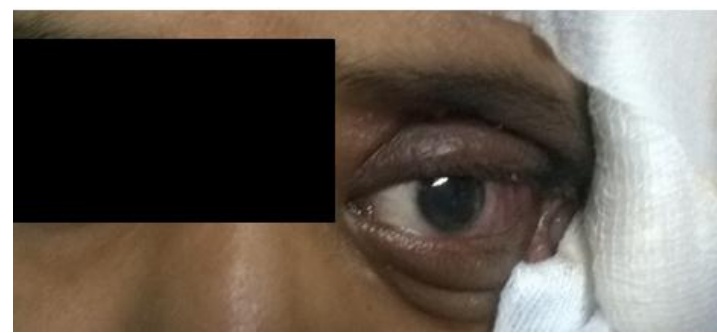

Fig. 1: Ill-defined lobulated soft tissue swelling in the left fronto-temporal region with intraorbital extension showing chemosed conjunctiva with purulent discharge

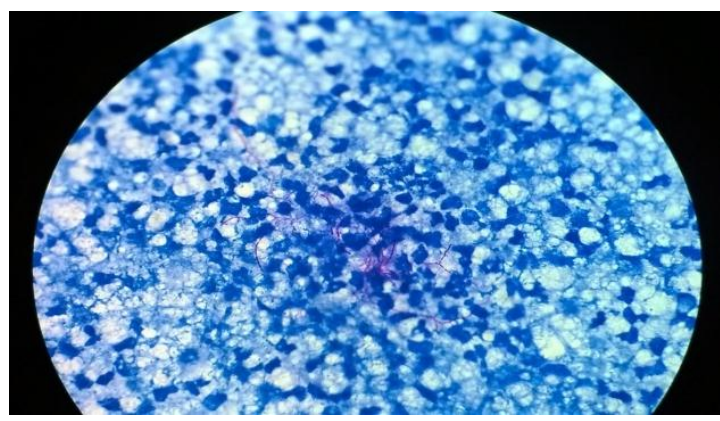

Fig. 2: Modified kinyoun's acid fast stain with $1 \%$ $\mathrm{H}_{2} \mathrm{SO}_{4}$ showing long acid fast bacilli in branching filamentous pattern with numerous polymorphonuclear leucocytes (PMNL)

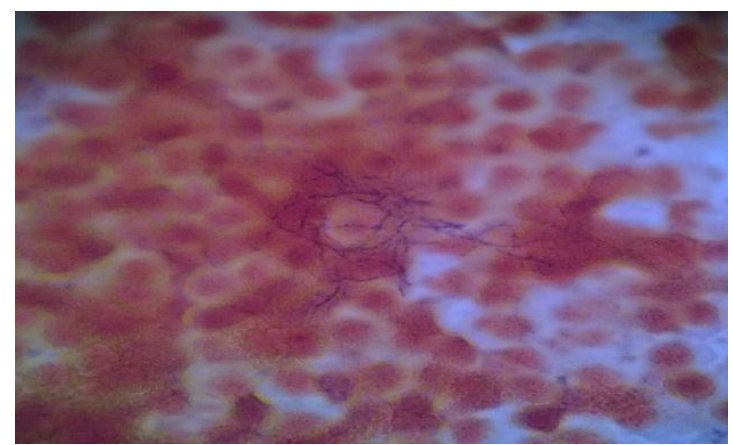

Fig. 3: Gram stain of pus showing long gram positive bacilli in branching filamentous pattern with numerous polymorphonuclear leucocytes (PMNL)

Based on the gram stain findings, the specimen was inoculated on blood agar, Sabourauds dextrose agar and Lowenstein Jensen (LJ) medium. Blood agar grew dry chalky white colonies after 72 hours of aerobic incubation with $5-10 \%$ of $\mathrm{CO}_{2}$. LJ medium also grew pale moist yellow colonies of nocardia after 6 days of aerobic incubation.

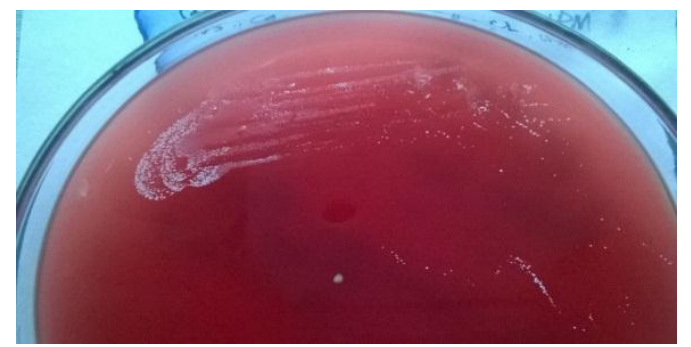

Fig. 4: Blood agar showing dry chalky white colonies of Nocardia asteroides after 72 hours of incubation

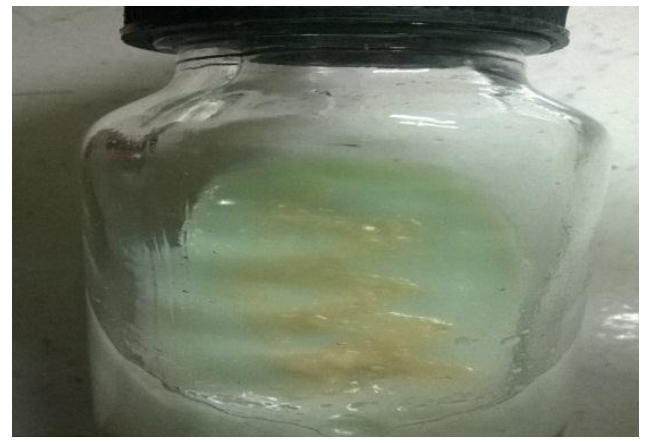

Fig. 5: Lowenstein Jesnsens medium showing yellow colonies of Nocardia asteroids

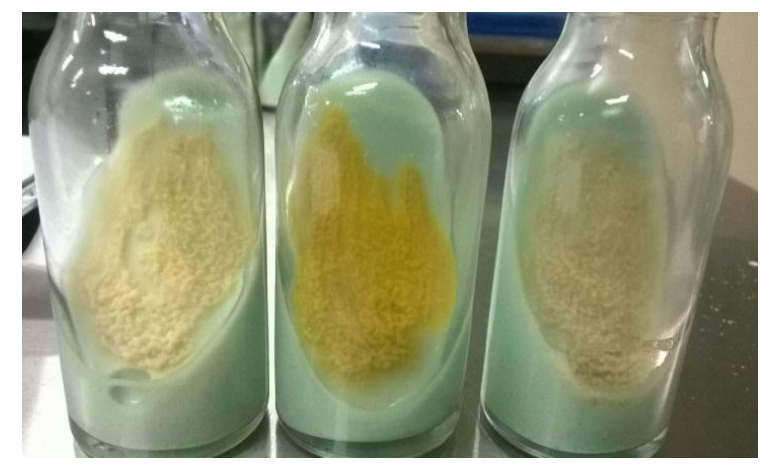

Fig. 6: Growth control on Lowenstein Jensen's medium with different species of mycobacteria

The recommended method for susceptibility testing of the aerobic actinomycetes (Nocardia species, Actinomadura species, Rhodococcus species, Gordona species, Tsukamurella species, Streptomyces species, etc.) is broth microdilution as per the NCCLS document (M24-A, Vol. 23 No. 18) M7-Methods for Dilution Antimicrobial Susceptibility Tests for Bacteria that Grow Aerobically. This standard provides protocols and related quality control parameters and interpretive criteria for the susceptibility testing of mycobacteria, Nocardia spp., and other aerobic actinomycetes. Drugs that are recommended for primary testing are: amikacin, amoxicillin-clavulanic acid, ceftriaxone, ciprofloxacin, clarithromycin, imipenem, linezolid,

IP International Journal of Medical Microbiology and Tropical Diseases, April-June, 2018;4(2):103-107 104 
minocycline, trimethoprim-sulfamethoxazole, and tobramycin. Secondary drugs that may be tested are cefepime, cefotaxime, doxycycline, gatifloxacin or moxifloxacin, and gentamicin. The minimum inhibitory concentrations (MIC) was read in $\mu \mathrm{g} / \mathrm{ml}$. It was determined in accordance with guidelines provided by CLSI breakpoints.

Table.1: Antimicrobial susceptibility pattern of Nocardia spp.

\begin{tabular}{|l|c|c|c|}
\hline \multicolumn{1}{|c|}{ Antibiotic } & MIC Range $(\boldsymbol{\mu g} / \mathbf{m l})$ & Observed MIC & Interpretation \\
\hline Cotrimoxazole & $<2 / 38->=4 / 76$ & $<2 / 16$ & Susceptible \\
\hline Vancomycin & $<=4$ & 0.5 & Susceptible \\
\hline Linezolid & $<=8$ & 1.0 & Susceptible \\
\hline Ciprofloxacin & $<=1->=4$ & 0.5 & Susceptible \\
\hline Amikacin & $<=8->=16$ & 2.0 & Susceptible \\
\hline Gentamicin & $<=4->=16$ & 1.0 & Susceptible \\
\hline Imipenem & $<=4->=16$ & 2.0 & Susceptible \\
\hline Cefotaxime & $<=8->=64$ & 8.0 & Susceptible \\
\hline Ceftriaxone & $<=8->=64$ & 8.0 & Susceptible \\
\hline
\end{tabular}

Based on antimicrobial susceptibility testing, cotrimoxazole (COT) was started (Bactrim DS twice a day) and ceftriaxone 2.5 grams intravenous infusion twice a day. Patient did not show any evidence of disseminated nocardiosis as microbiological examination other samples like blood; body fluids and sputum were negative for culture. The antimicrobials were continued for 3 weeks in oral and intravenous form following inpatient admission and patient improved clinically with subsidence of pain and swelling. After discharge, oral cotrimoxazole is prescribed for 3 months duration in double strength.

\section{Discussion}

Nocardiosis, caused by aerobic, gram-positive actinomycetes is an uncommon and serious infection affecting immunocompromised patients with HIV, cancer, patients undergoing organ transplantation and patients on long standing chemotherapy and systemic corticosteroids. Defective cell mediated immunity is the most important risk factor and approximately $65-70 \%$ of cases occurs individuals with compromised immune status. ${ }^{7}$ Nocardia asteroids is the most common organism implicated in human nocardiosis. Others like $N$. brasiliensis, $N$. nova, $N$. farcinica and $N$. transvalensis are also rarely implicated. ${ }^{8}$ It is a soil saprophyte, the mode of transmission is inhalation and commonly causes pulmonary infection by entering via the respiratory tract and person-to-person transmission is rare. ${ }^{9}$ The manifestations of nocardiosis can be solely pulmonary $(75-85 \%)$, cutaneous or neurological $(30 \%)$ but virtually any organ system may be involved in the disease. ${ }^{10}$ Disseminated nocardiosis is defined as when there is involvement of two or more noncontiguous organs, with or without involvement of central nervous system. The incidence of disseminated disease is 25 $40 \% .^{11}$ The risk of disseminated disease is greater among persons with deficient cell mediated immunity associated with malignancies like lymphoma, leukemia, bronchogenic and metastatic lung carcinomas, solid organ transplantation with high dose of immunosuppressive agents, systemic corticosteroid therapy or advanced AIDS disease with substantial reduction in CD4 count. CD4 count of less than 50 cells $/ \mathrm{mm}^{3}$ predisposes the patient to develop opportunistic infections. Severity and duration of neutropenia in cancer patients is known to develop opportunistic infections. Long standing neutropenia with absolute neutrophil count (ANC) less than 500 cells $/ \mathrm{mm}^{3}$ also plays a significant role to develop infections like nocardiosis. Pulmonary alveolar proteinosis and sarcoidosis are also known to be complicated by nocardiosis. ${ }^{12}$ The clinical manifestations in nocardiosis are relatively nonspecific depending on the site affected with a chronic course in almost $70 \%$ of patients before diagnosis. The following differentials like pneumonia, sarcoidosis, aspergillosis and other fungal infections, actinomycosis, tuberculosis, bronchogenic and metastatic lung carcinomas, lung abscess, and brain tumors should be kept in mind in suspected cases of nocardiosis. ${ }^{13}$ In our present case, the nocardial infection site is very unusual. The patient had described history of road traffic accident; probably this has led to the inoculation of organism at the traumatic site. Patient was not aware of her serological status at the time of hospital admission. Serological markers were done before FNAC as a routine protocol. The patient was found to be sero-positive for HIV antibodies and HIV-I RNA PCR. The diagnosis should always be based on demonstration of organism either by microscopy or culture from the affected site. Culture remains the gold standard for diagnosis. In suspected cases of nocardiasis, if $\mathrm{g}$ stain is showing long filamentous gram positive bacilli with branching pattern, all such specimens should be subjected to modified Kinyoun's acid fast staining with $1 \% \mathrm{H}_{2} \mathrm{SO}_{4}$ to rule out acid fastness. Nocardia are said to weakly acid fast. Based on gram stain findings, the specimen should be inoculated on blood agar, Loweinstein Jensens (LJ) 
medium and SDA (to rule out fungal elements). Nocardia is a relatively slow growing actinomycete and colonies may take around 2 to 3 weeks to appear on $\mathrm{LJ}$ medium or to take their characteristic appearance. On blood agar growth is relatively rapid with appearance of dry chalky white colonies within a week. All the cultures should be incubated for at least 3 weeks before being discarded as no growth. Concentration and decontamination of clinical specimens should be followed routinely before processing on culture media to avoid growth of other contaminating organisms as it may be a polymicrobial infection. The gram stain and modified acid fast stain findings should be informed to the clinicians to initiate early antimicrobial therapy as the culture results will be prolonged. Molecular techniques like polymerase Chain Reaction (PCR), 16s rRNA sequence analysis, Restriction Fragment Length Polymorphism (RFLP) can also be used in diagnosis based on the facilities available. Dissemination of nocardiosis in patients with HIV is not infrequent. A careful history should be obtained and a thorough physical examination is performed along with cultures from other body sites is taken if clinically indicated. Signs and symptoms suggestive of brain involvement, CT or MRI of the head, with or without contrast should be undertaken along with CSF cultures. It is essential to perform antimicrobial susceptibility testing in the clinical specimens isolating nocardia species because of the need for multiple antibiotics in severe disseminated infections and variable susceptibility of these organisms to different antimicrobials as per CLSI 2003 guidelines for nocardial antimicrobial susceptibility testing which describes broth micro dilution as a standard and reliable method. Cotrimoxazole (Trimethoprimsulfamethoxazole) is the drug of choice which can be given alone or in combination with other drugs like imipenem, meropenem, amikacin, gentamicin, vancomycin, linezolid, third-generation cephalosporins and minocycline in serious cases based on the MIC values. It essential to use synergistic drug combination therapy to avoid emergence of drug resistance and relapse. Clinical studies with combination of drugs like imipenem-amikacin, imipenem-cefotaxime and amikacin-cefotaxime have demonstrated in vitro synergy supported with clinical evaluation (in vivo). ${ }^{14}$ In localized disease, the therapy should continued for at least 3-6 months. In HIV patients with disseminated disease it is recommended to administer the drugs for one year or more depending upon severity of infection and the host immune status. It is also advisable to serially monitor the CD4 Lymphocyte count and HIV RNA viral load to determine the duration of treatment. Disseminated nocardiosis has got poor prognosis. Mortality rate may be as high as $85 \%$ in immunocompromised hosts with advanced HIV. ${ }^{15}$ Therefore early diagnosis and prompt initiation of antimicrobial therapy in a suspected cases of nocardiosis will help to improve the clinical outcomes.

\section{Conclusion}

Nocardiosis is an important cause of opportunistic infection in immunosuppressed patients. With increasing number of immunocompromised conditions like HIV, cancer, patients undergoing organ transplantations with long term systemic glucocorticoid therapy, diagnosis of Nocardia infections should always be kept in mind as it can present with nonspecific symptoms and can mimic with other diseases like pneumonia, tuberculosis, aspergillosis and brain and musculoskeletal neoplasms. Sulfonamides are the drugs of choice but other drugs like imipenem, vancomycin, linezolid, amikacin and minocycline were found to be very effective, in vitro, against most Nocardia species. It advisable to use synergistic drug combination to prevent emergence of drug resistance and relapse. Early recognition and prompt treatment usually results in complete cure especially in immunosuppressed patients with disseminated nocardiosis. Rapid and accurate diagnosis of nocardiosis resulted in a favorable outcome in a case presenting as a retro-orbital neoplasm.

Acknowledgements: We are thankful to the Department of Microbiology, Tata Memorial Hospital, Mumbai, India- 400012.

\section{Conflict of Interest: None}

Funding: No source of funding has been received

\section{References}

1. Saubolle MA, Sussland D. Nocardiosis: review of clinical and laboratory experience. J Clin Microbiol 2003;41:4497-4501.

2. Lerner PL. Nocardiosis. Clin Infect Dis 1996;22:891903.

3. McNeil MM, Brown JM. The medically important aerobic Actinomycetes: epidemiology and microbiology. Clin Microbiol Rev 1994;7:357-417.

4. Brown-Elliott BA, Brown JM, Conville PS, Wallace RJ Jr. Clinical and laboratory features of the Nocardia spp. based on current molecular taxonomy. Clin Microbiol Rev 2006;19:259-282.

5. Beaman BL, Beaman L. Nocardia species: host-parasite relationships. Clin Microbiol Rev 1994;7:213-264.

6. Kageyama A, Yazawa K, Ishikawa J, Hotta K, Nishimura K, Mikami Y. Nocardial infections in Japan from 1992 to 2001, including the first report of infection by Nocardia transvalensis. Eur J Epidemiol 2004;19:383-389.

7. Frazier A, Rosenow E, Roberts G. Nocardiosis: a review of 25 cases occurring during 24 months. Mayo Clin Proc 1975;50:657-63.

8. Wadhwa V, Rai S, Kharbanda P, Kabra S, Gur R, Sharma VK. A fatal pulmonary infection by nocardia brasiliensis. Indian Journal of Medical Microbiology 2006;24:63-4.

9. Palmer DL, Harvey RL, Wheeler JK. Diagnostic and therapeutic considerations in Nocardia asteroides infection. Medicine 1974;53:391-401.

10. Sud S, Buxi TBS, Anand I, Rohatgi A. Case series: nocardiosis of the brain and lungs. Indian J Radiol Imaging 2008;18:218-21. 
11. Wakhlu A, Agarwal V, Dabadghao S, Prasad KN,

Nityanand S. Nocardiosis in patients of chronic idiopathic thrombocytopenic purpura on steroids. J Assoc

Physicians India 2004;52:501-3.

12. Jastrzembski SA, Teirstein AS, Herman SD, Depalo LR, Lento PA. Nocardiosis presenting as an anterior mediastinal mass in a patient with sarcoidosis. The Mount Sinai Journal of Medicine 2002;69:350-3.

13. Hwang JH, Koh WJ, Suh GY, Chung MP, Kim H, Kwon $\mathrm{OJ}$ et al. Pulmonary nocardiosis with multiple cavitary nodules in the immunocompromised HIV-negative patient. Internal Medicine 2004;43:852-54.

14. Gombert ME, Aulicino TM, du Bouchet L, Silverman GE, Sheinbaum WM. Therapy of experimental cerebral nocardiosis with imipenem, amikacin, trimethoprim sulfamethoxazole, and minocycline. Antimicrob Agents Chemother 1986;30:270-3.

15. Lederman ER, Crum NF. A case series and focused review of nocardiosis: clinical and microbiologic aspects. Medicine 2004;83:300-13. 